\title{
Optical tests of Galileo's lenses
}

\author{
Vincenzo Greco, Giuseppe Molesini and Franco Quercioli
}

\section{The science museum in Florence has two telescopes and a single lens attributed to Galileo. Tests conducted with modern interferometric equipment show that Galileo was able to obtain nearly perfect optical quality.}

ACCORDING to biographies, Galileo is supposed to have made several telescopes, purchasing some lenses and polishing others himself, yet only the optical apparatus now in Florence, collected and handed down by the Medici family ${ }^{1}$, appears to have survived. The authenticity of the telescope tubes seems certain, but some doubts remain about the lenses, apart from the single lens, which was used in the discovery of the Medici stars.

The Medici collection was examined in $1923^{2,3}$, but we have now taken the telescopes apart and tested them with state-of-the-art optical equipment. Analysis of the optical quality of Galileo's lenses is of interest for understanding both the development of optical technology and the observational capabilities of early astronomers. Before Galileo's time, observations were made with the naked eye, for which the resolution is about 1 arc minute. Lenses were used only as eyeglasses or as magnifying lenses, for which applications the poor glass purity and optical figure then feasible were nevertheless adequate.
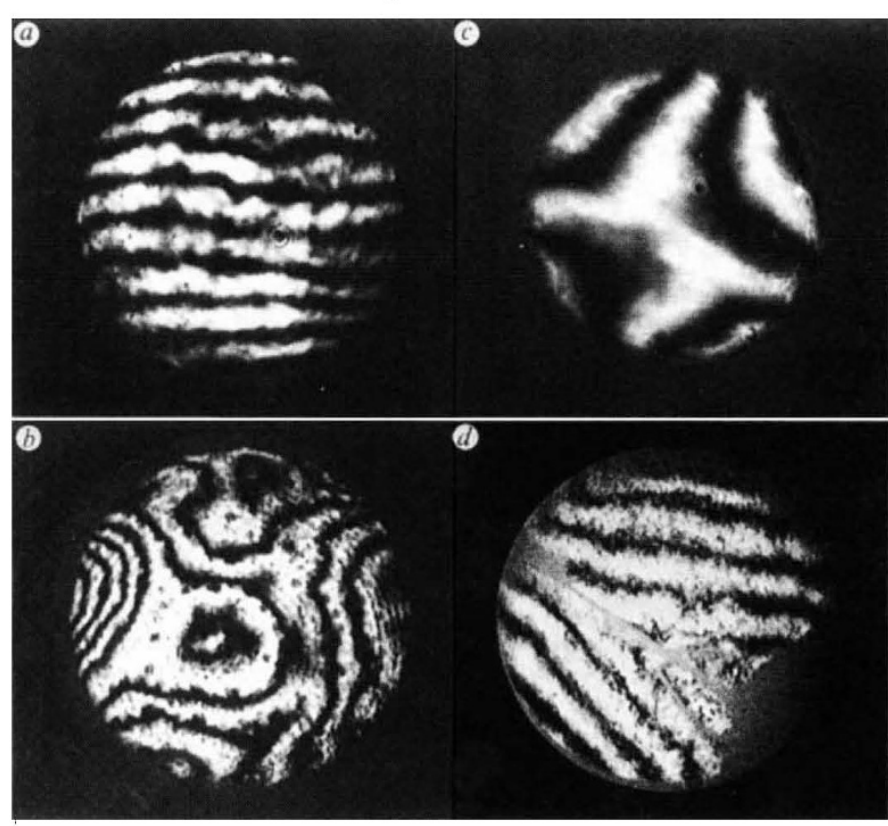

Fizeau fringe patterns of the optical elements of Galileo's telescopes at 633 $\mathrm{nm}$. The fringe maps show the deviation of the wavefront from a sphere or a plane. For reference, a diffraction-limited wavefront produces no fringes, or straight and equaliy spaced fringes if some tilt is added. a, Double-pass interferogram of objective I, folded with a reference mirror. Deviations from straight fringes are of the order of half a pitch, meaning a departure from the ideal wavefront of the order of a quarter of a wavelength. $b$. Reflection interferogram of the concave surface of eyepiece I. Fringes are highly irregular $c$, Reflection interferogram of the plane surface of objective II. The quadrant fringes show astigmatism. $d$, Double-pass interferogram of the single lens.

NATURE - VOL 358 - 9 JULY 1992 The focal
We have examined Galileo's optics with modern methods. We call the telecopes I (paper coated, longer tube) and optical configuration is based on a positive objective and a negative eyepiece

OPTICAL DATA OF GALILEO'S LENSES

\begin{tabular}{|c|c|c|c|c|c|}
\hline $\begin{array}{l}\text { Front } \\
\text { radius }\end{array}$ & $\begin{array}{l}\text { Back } \\
\text { radius }\end{array}$ & $\begin{array}{l}\text { Central } \\
\text { thickness }\end{array}$ & $\begin{array}{l}\text { Full } \\
\text { diameter }\end{array}$ & $\begin{array}{l}\text { Aperture } \\
\text { diameter }\end{array}$ & $\begin{array}{l}\text { Focal } \\
\text { length }\end{array}$ \\
\hline 2,700 & 950 & 2.5 & 51 & 26 & 1,330 \\
\hline plane & $48.5\left(^{*}\right)$ & 3.0 & 26 & 11 & -94.0 \\
\hline $535\left(^{*}\right)$ & Plane & 2.0 & 37 & 16 & 980 \\
\hline $51.5\left(^{*}\right)$ & $51.5\left(^{*}\right)$ & 1.8 & 22 & 16 & -47.5 \\
\hline 940 & 12,000 & 4.0 & 58 & 38 & 1,710 \\
\hline
\end{tabular}
calculate that the refractive relative apertures of the objectives are $f / 51$ for telescope I, $f / 61$ for telescope II and $f / 45$ for the single lens. The magniopes is 14 for I and 21 for II.

We used a 633nm digital phaseshift Fizeau interferometer to study the regularity of the optical surfaces and the wavefront distortion in transmission. Typical fringe patterns are shown in the figure. As far as regularity is concerned, the quality of the objective lenses is far better than the quality of the eyepieces. But because the used diameter per field angle at the eyepiece is much smaller than the clear aperture of the objective, the effect of the lower quality of the eyepieces is negligible. It is surprising that the plane surfaces of eyepiece I and objective II ( $c$ in the figure) are really plane to a fraction of a wave, although flatness of these surfaces is not required in terms of image quality. (Polishing a surface to such flatness is not trivial even for today's crown

\section{glasses.)}

A further observation comes from the appearance of the concave surface of eyepiece I $(b)$. In addition to the interference fringes, a pattern of ring shadows appears, as if the surface had traces of a turning process. The wavefront distortion of objective I is very small $(a)$. The best quality belongs to the single lens, which can be considered as nearly diffraction-limited $(d)$. According o the Rayleigh criterion, its resolution at $633 \mathrm{~nm}$ is of the order of 3 arcseconds. Of course, the optical performance of the telescopes is degraded for several reasons, mainly chromatic aberration. Computer simulations taking dispersion into account lead to estimates of only 10-20 arcseconds resolution over the visible spectrum.

Altogether, our tests of the lenses (made 350 years after Galileo's death) show that they are polished to a good spherical shape, and the presence of proper apertures on the objectives also shows Galileo's awareness of the need to tune the optical performance. As a result, although affected by intrinsic chromatic aberration, at single wavelength the telescopes are nearly diffraction-limited, that is, optically perfect.

Vincenzo Greco, Giuseppe Molesini and Franco Quercioli are at the Istituto Nazionale di Ottica, Largo E. Fermi 6, Firenze 50125, Italy.

ACKNOWLEDGEMENTS. We thank P. Galluzzi and M. Miniati for discussions, and the tstituto e Museo di Storia della Scienza for permission to publish the material in this paper.

1. Bonelli, M. L. in Proc. 1964 Symp. Galileo 125-127 (Marzocco, Florence, 1967)

2. Abetti, G. L'Universo, 4, 685-692 (1923)

3. Ronchi, V. L'Universo, 4, 791-804 (1923) 